\title{
Cardiac left heart morphology and function in newborns with intrauterine growth restriction: relevance for long-term assessment
}

\author{
Gabriela C. Zaharie1, Monica G. Hăşmăşanu1, Ligia Blaga1, Melinda Matyas¹, \\ Daniel Mureșan², Sorana D. Bolboacă ${ }^{3}$
}

${ }^{1}$ Department of Neonatology, ${ }^{2}$ Department of Obstetrics and Gynecology, ${ }^{3}$ Department of Medical Informatics and Biostatistics, „Iuliu Haţieganu” University of Medicine and Pharmacy, Cluj-Napoca, Romania

\begin{abstract}
Aim: To asses the cardiac morphology and functional changes specific for newborns from intrauterine growth restriction (IUGR) pregnancies. Material and method: A cohort of IUGR infants were evaluated by serial echocardiographies at delivery and at the first and six months follow-ups. IUGR newborn delivery status was compared to that of newborns in the control group according to gestational age (AGA). Results: Left heart measurements were significantly lower in IUGR newborns compared to AGA babies. Left ventricular size increased at follow-up inthe IUGR group $(\mathrm{p}<0.05)$. Systolic dysfunction (the myocardial performance index (MPI) $>0.47$ ) was identified in $40 \%$ of the neonates in the IUGR group (16/40), respectively $4.76 \%$ in the control group. IUGR neonates had a significantly increased proportion of systolic malfunction ( $p=0.004)$. Conclusion: IUGR patients had reduced left ventricle dimensions compared to AGA babies. The MPI stands out as a marker of left heart function in newborns. Systolic dysfunction was a hallmark of the cardiac adaptation in IUGR neonates.

Keywords: birth weight; intrauterine growth restriction; left ventricular morphology; myocardial performance index
\end{abstract}

\section{Introduction}

Intrauterine growth restriction (IUGR) is defined as a fetal growth rate below the normal growth potential according to the race and gender of a certain infant $[1,2]$. Small for gestational age (SGA) represents birthweight less than two standard deviations below the mean or birth weight under the $10^{\text {th }}$ percentile on population-specificnomograms for a specified gestational age [2]. Both entities are clearly defined, but sometimes these two terms are used interchangeably [3]. IUGR is diagnosed prenatally based on the ultrasound measurement of fetal bi-

Received 20.08.2018 Accepted 28.11.2018

Med Ultrason

2019, Vol. 21, No 1, 62-68

Corresponding author: Monica G. Hășmășanu

Department of Neonatology

3-5 Clinicilor Street,

400006 Cluj-Napoca, Romania

E-mail: monica.hasmasanu@gmail.com

Phone: +40741985974 ometry under the $10^{\text {th }}$ centile for the gestational age with associated umbilical artery and/or middle cerebral artery colour Doppler abnormalities [3,4].

Fetal distress, defined as progressive fetal hypoxia and/or acidemia secondary to inadequate fetal oxygenation, is a term used to indicate changes in the fetal heart pattern, reduced fetal movement and fetal growth restriction. Chronic hypoxia can lead to brain sparing in fetal life with prefferent flow towards vital organs: brain, heart and adrenal glands. The cardiac morphology and functional analysis of IUGR fetuses in utero demonstrated changes in the inventricular geometry [5-8]. Fetuses with IUGR present systolic and/or diastolic dysfunction [9] with changes in the myocardial relaxation and atrial contraction patterns, increased the E/A ratio and the increased myocardial performance index [5]. These changes occur in the early fetal period in response to a certain trigger and can persist after birth causing heart failure and vascular remodeling [10]. Infants with IUGR have persistent postnatal cardiac morphological changes such as cardiac septal hypertrophy or left ventricle dilatation 
[11-13]. However, functional changes have not always been documented using conventional echocardiographic parameters [13,14].

The assessment of systemic flow in the first days postnatally is difficult due to the persistence of fetal shunts [15]. Left ventricular functions is assessed in the neonatal period by the M-mode measurement of the ejection fraction (EF) and shortening fraction (SF) in the long parasternal axis. The SF tends to change with changing cavity dimensions, while paradoxical motion oft he septal wall is observed in cases with persistent pulmonary hypertension [15]. The EF may be a better tool but it is closely influenced by the ventricular preload [15]. The myocardial performance index has been proposed as a parameter in the assessment of systolic and diastolic performance but it can be influenced by preload and afterload [16]. Normal values of MPI for healthy term newborns range from 0.25 to 0.38 but few studies assess its reproducibility [17].

In order to find the echocardiographic markers of heart function impairement in IUGR newborns at delivery and for short term evolution, we assessed the cardiac morphology and function in IUGR and appropriate for gestational age (AGA) newborns, comparatively.

\section{Material and methods}

This is a longitudinal prospective cohort study conducted in a third level neonatology center between June 2014 and June 2015 on 40 neonates with IUGR and 21 newborns with birthweight AGA. The Ethical Committee of the Iuliu Haţieganu University of Medicine and Pharmacy Cluj-Napoca approved the study. Inclusion of patients in the study groups was made after the signed agreement from their mothers was obtained.

IUGR was defined as fetal development below the 10th centile associated with Doppler changes on the umbillical artery or/and middle cerebral artery. AGA was defined as babies born from uncomplicated pregnancies. Control patients were matched for gestational age with IUGR patients. Exclusion criteria for both study populations were represented by confirmed chromosomopathies and the presence of fetal cardiac malformations.

The initial newborn clinical examination was carried out in the first day of life and included recordings of anthropometric data such as weight $(\mathrm{g})$, length $(\mathrm{cm})$ and head circumference $(\mathrm{cm})$ evaluated according to the preterm growth Fenton charts [18].

Echocardiographic evaluation of the left heart was performed after delivery by a single investigator using a GE LOGIQe ultrasound machine with a GE 6S-RS pediatric, cardiac sector transducer of 2.5-8.0 MHz. The val- ues were reported newborn nomograms and the Z-score was defined as the number of standard deviations from the population mean [19]. Evaluation was made in the parasternal long axis section, in M-mode (fig1) following the guidelines of the American Society of Echocardiography (ASE), the European Association of Echocardiography (EAE) and the Association for European Paediatric Cardiologists (AEPC) [20].

Left ventricle measurements were calculated by the Haycock formula using Z-scores and adjusted according to the body surface area [21]. Measurements respected the recommendations of the Council on Pediatric and Congenital Heart Disease of the American Society of Echocardiography [21,22].

For the assessment of cardiac function, the following 3 parameters were used: a) diastolic functiondefined by the ratio to early filing (E wave) and atrial contraction (A wave) at the level of the mitral valve (E/A); b) systolic function; and c) systolic and diastolic function: MPI = (ab)/b where a represents the time between the ending and beginning of the transmitral diastolic flow, and $\mathrm{b}$ meaning the time between the beginning to the end of aortic systolic flow (fig 2).

The IUGR cohort received additional follow-up echocardiographies at one month and 6 months age respectively.

\section{Statistical analysis}

The data collected were analysed using the Statistica software version 8 (StatSoft Inc., USA). Continuous data were summarized as mean \pm standard deviation whenever data proved to be normally distributed (Shapiro-Wilk test). The association in the two by two contingency table was tested with Fisher's exact test. The differences between groups were tested with the Z-test for proportions on qualitative data. Comparisons between groups on continuous data were tested with the t-test after checking for the distribution of data. The paired t-test was used to compare the baseline and follow-up values of the IUGR group. The Pearson correlation coefficient was used to assess the correlation between anthropometric and echocardiographic data. A p-value below 0.05 was considered statistically significant.

\section{Results}

We analyzed 40 growth restricted infants compared to 21 appropriate for the gestational age controls. The gender distribution and anthropometric data are presented in Table I.

The size of the interventricular septum in systole (IVSs), the end diastolic size of the left ventricle (LVDD) and the size of the posterior wallof the left ventricle 
Table I. Baseline anthropometric data of the studied groups

\begin{tabular}{lllll}
\hline Characteristic & All $(\mathbf{n}=\mathbf{6 1})$ & IUGR $(\mathbf{n}=\mathbf{4 0})$ & Control $(\mathbf{n}=\mathbf{2 1})$ & p-value \\
\hline Gestational age & $35.85(3.06)$ & $35.83(3.17)$ & $35.90(2.91)$ & $0.923^{\mathrm{a}}$ \\
Caesarean delivery (\%) & 67.21 & 80.00 & 42.86 & $0.003^{\mathrm{b}}$ \\
Gender: Girls (\%) & 57.38 & 62.50 & 47.62 & $0.263^{\mathrm{b}}$ \\
Birth weight (g) & $2014(738)$ & $1794(606)$ & $2643(737)$ & $<0.001^{\mathrm{a}}$ \\
Length (cm) & $46(6)$ & $45(6)$ & $49(4)$ & $0.012^{\mathrm{a}}$ \\
Cranial perimeter $(\mathrm{cm})$ & $31(3)$ & $30(3)$ & $32(1)$ & $0.046^{\mathrm{a}}$ \\
\hline
\end{tabular}

$\mathrm{a}=\mathrm{t}$-test; $\mathrm{b}=\mathrm{Z}$ test for proportions; IUGR $=$ intrauterine growth restriction; $\mathrm{m}(\mathrm{SD})=$ mean (standard deviation)

(LVPW) proved to be significantly smaller in the IUGR group compared to the AGA group (Table II) at delivery.

According to the Z-scores, the size of the interventricular septum in diastole (IVSd) and LVPW in systole had pathological values in two subjects in the IUGR group $(5 \%)$, both cases with measurements over two standard deviations. Statistically significant differences between the systolic function evaluated by MPI were identified between groups with higher values in the IUGR group.

Systolic dysfunction (MPI>0.47) was identified in $40 \%$ of the neonates in the IUGR group (16/40), respectively $4.76 \%$ of subjects in the control group $(1 / 21)$. There was a significant association between systolic dysfunction reflected by MPI and the occurence of IUGR ( $\mathrm{p}$ $=0.004$ ).

Follow-up cardiac evaluations of the IUGR group patients was performed at one month in 36 subjects and at six monthsin 33 subjects. Two subjects were deceased and two were lost until theone-month follow-up and three more until six months.

At re-evaluation, $8(22.22 \%)$ patients in the IUGR group had smaller or equal size interventricular septum measured in systole compared to delivery measurements.
The size of the left ventricle increased from delivery to the one month and six months measurements respectively. All subjects had normal Z-score values at the six months follow-up (Table III).

Table II. Left heart morphometric and function characteristics: comparison between groups

\begin{tabular}{llll}
\hline Characteristics & $\begin{array}{l}\text { IUGR } \\
(\mathbf{n}=\mathbf{4 0})\end{array}$ & $\begin{array}{l}\text { Control } \\
(\mathbf{n}=\mathbf{2 1})\end{array}$ & p-value \\
\hline IVSs $(\mathrm{mm})$ & $5.08(1.14)$ & $5.74(1.32)$ & 0.048 \\
IVSd (mm) & $3.29(0.83)$ & $3.63(0.69)$ & 0.114 \\
LVDD (mm) & $14.10(2.38)$ & $16.20(2.30)$ & 0.002 \\
LVSD (mm) & $9.90(2.19)$ & $10.63(1.64)$ & 0.186 \\
LVPW (mm) & $2.62(0.53)$ & $2.92(0.55)$ & 0.041 \\
FS (\%) & $29.70(10.43)$ & $33.81(9.54)$ & 0.138 \\
E/A & $1.05(0.28)$ & $1.00(0.24)$ & 0.442 \\
MPI & $0.44(0.10)$ & $0.37(0.07)$ & 0.008 \\
\hline
\end{tabular}

The results are expressed as mean (standard deviation). IUGR $=$ intrauterine growth restriction; IVSs = Interventricular septum diameter in systole; IVSd = Interventricular septum diameter in diastole; LVDD = left ventricular end-diastolic dimension; $\mathrm{LVSD}=$ left ventricular end-systolic dimension; LVPW = left ventricular posterior wall diameter in systole; FS $=$ Fractional shortening; $\mathrm{E} / \mathrm{A}=$ Mitral inflow Doppler velocities at early (E) and late (A) diastole, $\mathrm{E} / \mathrm{A}$ ratio; $\mathrm{MPI}=$ myocardial performance index

Table III. Cardiac morphometric featuresin evolution on the intrauterine growth restriction group

\begin{tabular}{|c|c|c|c|c|c|c|}
\hline & \multirow{2}{*}{$\begin{array}{l}\text { Baseline } \\
(\mathrm{n}=40)\end{array}$} & \multirow{2}{*}{$\begin{array}{l}1 \text { month } \\
(\mathrm{n}=36)\end{array}$} & \multirow{2}{*}{$\begin{array}{l}6 \text { months } \\
(n=33)\end{array}$} & \multicolumn{3}{|c|}{ p-value } \\
\hline & & & & $\begin{array}{l}\text { baseline } v s \\
1 \text { month }\end{array}$ & $\begin{array}{l}\text { baseline } v s \\
6 \text { months }\end{array}$ & $\begin{array}{l}1 \text { month } v s \\
6 \text { months }\end{array}$ \\
\hline IVSs (mm) & $5.19(1.15)$ & $5.75(0.99)$ & $6.63(0.93)$ & 0.001 & $<0.001$ & $<0.001$ \\
\hline IVSd (mm) & $3.33(0.83)$ & $3.90(0.64)$ & $4.84(0.53)$ & $<0.001$ & $<0.001$ & $<0.001$ \\
\hline IVSd Z score & $0.29(1.21)$ & $0.77(0.85)$ & $0.84(0.35)$ & 0.020 & 0.026 & 0.464 \\
\hline LVDD (mm) & $14.29(2.41)$ & $16.15(2.15)$ & $19.6(1.67)$ & $<0.001$ & $<0.001$ & $<0.001$ \\
\hline LVDD Z-score & $-0.87(1.16)$ & $-0.57(0.91)$ & $-1.05(0.48)$ & 0.202 & 0.340 & 0.026 \\
\hline LVSD (mm) & $10.06(2.19)$ & $11.24(2.20)$ & $19.6(1.67)$ & $<0.001$ & $<0.001$ & $<0.001$ \\
\hline LVSD Z-score & $-0.13(1.16)$ & $0.22(1.08)$ & $-0.43(0.84)$ & 0.037 & 0.057 & $<0.001$ \\
\hline LVPW (mm) & $2.65(0.53)$ & $3.56(0.63)$ & $4.37(0.61)$ & $<0.001$ & $<0.001$ & $<0.001$ \\
\hline LVPW Z score & $0.10(1.03)$ & $1.24(0.99)$ & $1.02(0.69)$ & $<0.001$ & $<0.001$ & 0.039 \\
\hline
\end{tabular}

The results are expressed as mean (standard deviation). IUGR = intrauterine growth restriction; IVSs = Interventricular septum diameter in systole; IVSd = Interventricular septum diameter in diastole; LVDD = left ventricular end-diastolic dimension; LVSD = left ventricular endsystolic dimension; LVPW = left ventricular posterior wall diameter in systole; $\mathrm{n}=$ number of subjects 
Fourteen subjects with IUGR (38.89\%) had MPI values higher than or equal to those measured at one month postnatally. MPI values recorded at six months were lower than those recorded at birth (Table IV, fig 3).

Results regarding the correlation between gestational age at delivery, birth weight, length and morpho-functional assessment of the left heart at 1 month and six months are depicted in Table V.

\section{Discussions}

This study evaluated the morphological and functional changes of the left heart in IUGR newborns over a 6 months follow-up period. Our research provides direct clinical evidence that growth restricted neonates show changes in cardiac morphology and subclinical cardiac dysfunction. These are probably part of a remodelling process in the attempt to maintain circulatory requirements. Overall, this could be a mechanistic pathway for the association between fetal growth impairement and cardiovascular disease.

Our study aimed to identify those parameters which describe and announce cardiac change in order to identify patients that might be at risk for persistent cardiac abnormalities. IUGR neonates had smaller anthropometric measurements compared to AGA infants, as reported by previous researches $[14,25]$. The condition was more frequent in female newborns in our study $(p<0.05)$, consistent with literature findings [26]. IUGR is frequently an adaptive response of the fetus to vascular injury (increased placental impedance). The sequence of events triggered by hypoxia begins with peripheral vasoconstriction and brain sparing to maintain blood supply to vital organs, followed by myocardial malfunction in more advanced stages of fetal distress. Left heart measurements performed in IUGR patients and controls showed lesser left ventricle parameters compared to AGA babies.

Since vaginal delivery could be a challenge for an IUGR fetus, the route of delivery is operative for the majority of cases. Surgery may be elective - based of altered fetal ultrasound parameters (as a sign of chronic fetal distress) or emmergency in case of pathological cardiotocographic traces (acute fetal distress) [23,24]. However, insufficient data recommend the $\mathrm{C}$-section delivery for low birth weight newborns [25].

Ecocardiographic ultrasound measurements performed at delivery in our study groups identified lower values for all the parameters characterizing the left ventricle morphology and function in newborns suffering from chronic hypoxic injury. This supports the findings of previous studies conducted on cohorts of growth restricted infants $[27,28]$. Some authors found a direct correlation between the extent of morphologic change and the severity of IUGR [11].

Table IV. Evolution of the left ventricular function in the intrauterine growth restriction group

\begin{tabular}{llllll}
\hline & baseline & 1 month & 6 months & \multicolumn{2}{c}{ p-value } \\
\cline { 4 - 6 } & & & & baseline vs1 month & 1 month vs 6 months \\
\hline FS & $29.48(10.40)$ & $30.20(11.24)$ & $37.37(7.68)$ & 0.665 & $<0.001$ \\
E/A & $1.06(0.28)$ & $1.03(0.21)$ & $1.19(0.17)$ & 0.436 & $<0.001$ \\
MPI & $0.44(0.11)$ & $0.37(0.08)$ & $0.28(0.04)$ & 0.002 & $<0.001$ \\
\hline
\end{tabular}

The results are expressed as mean (standard deviation). $\mathrm{FS}=$ Fractional shortening; $\mathrm{E} / \mathrm{A}=$ Mitral inflow Doppler velocities at early $(\mathrm{E})$ and late (A) diastole; MPI = myocardial performance index

Table V. The correlation between gestational age, birth weight and birth length with cardiac morphological and functional features in the intrauterine growth restriction group

\begin{tabular}{lllllll}
\hline & \multicolumn{2}{c}{ Gestational age (weeks) } & \multicolumn{2}{c}{ Birth weight (g) } & \multicolumn{2}{c}{ Birth length (cm) } \\
\cline { 2 - 7 } & $\mathbf{R}(\mathbf{p}) \mathbf{1 ~} \mathbf{~ m}$ & $\mathbf{R}(\mathbf{p}) \mathbf{6 ~ \mathbf { ~ m }}$ & $\mathbf{R}(\mathbf{p}) \mathbf{1 ~ m}$ & $\mathbf{R}(\mathbf{p}) \mathbf{6 ~ \mathbf { ~ m }}$ & $\mathbf{R}(\mathbf{p}) \mathbf{1 m}$ & $\mathbf{R}(\mathbf{p}) \mathbf{6 ~ m}$ \\
\hline LVDD $(\mathrm{mm})$ & $0.589(<0.001)$ & $0.467(0.006)$ & $0.646(<0.001)$ & $0.525(0.002)$ & $0.646(<0.001)$ & $0.411(0.018)$ \\
LVDS (mm) & $0.438(0.008)$ & $0.242(0.174)$ & $0.491(0.002)$ & $0.337(0.055)$ & $0.491(0.002)$ & $0.242(0.175)$ \\
LVPW (mm) & $0.312(0.064)$ & $0.431(0.012)$ & $0.365(0.029)$ & $0.484(0.004)$ & $0.365(0.029)$ & $0.299(0.091)$ \\
E/A & $0.562(<0.001)$ & $0.428(0.013)$ & $0.562(<0.001)$ & $0.408(0.018)$ & $0.562(<0.001)$ & $0.437(0.011)$ \\
IVSs (mm) & $0.317(0.059)$ & $0.336(0.056)$ & $0.402(0.015)$ & $0.418(0.015)$ & $0.402(0.015)$ & $0.425(0.014)$ \\
IIVSd (mm) & $0.318(0.059)$ & $0.402(0.020)$ & $0.337(0.044)$ & $0.459(0.007)$ & $0.337(0.044)$ & $0.358(0.041)$ \\
MPI & $0.037(0.831)$ & $0.062(0.731)$ & $0.173(0.312)$ & $0.036(0.842)$ & $0.173(0.312)$ & $0.042(0.817)$ \\
FS (\%) & $-0.005(0.976)$ & $0.005(0.98)$ & $-0.032(0.854)$ & $-0.07(0.701)$ & $-0.032(0.854)$ & $-0.017(0.925)$ \\
\hline
\end{tabular}

$1 \mathrm{~m}=$ one month; $6 \mathrm{~m}$ = six months; $\mathrm{R}=$ Pearson's correlation coefficient; $(\mathrm{p})=\mathrm{p}$-value; IVSs $=$ Interventricular septum diameter in systole; IVSd $=$ Interventricular septum diameter in diastole; $\mathrm{LVDD}=$ left ventricular end-diastolic dimension; LVSD $=$ left ventricular end-systolic dimension, LVPW = left ventricular posterior wall diameter in systole, $\mathrm{FS}=$ Fractional shortening; $\mathrm{E} / \mathrm{A}=$ Mitral inflow Doppler velocities at early (E) and late (A) diastole; MPI = myocardial performance index 


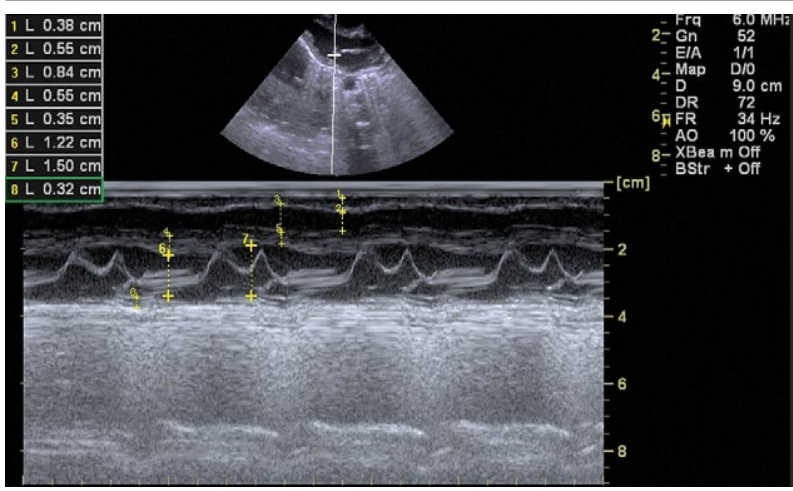

Fig 1. Morphometric evaluation of the left ventricle

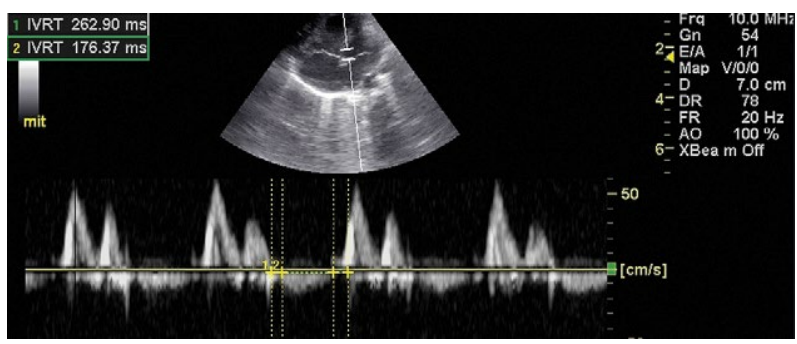

Fig 2. Evaluation of the myocardial performance index

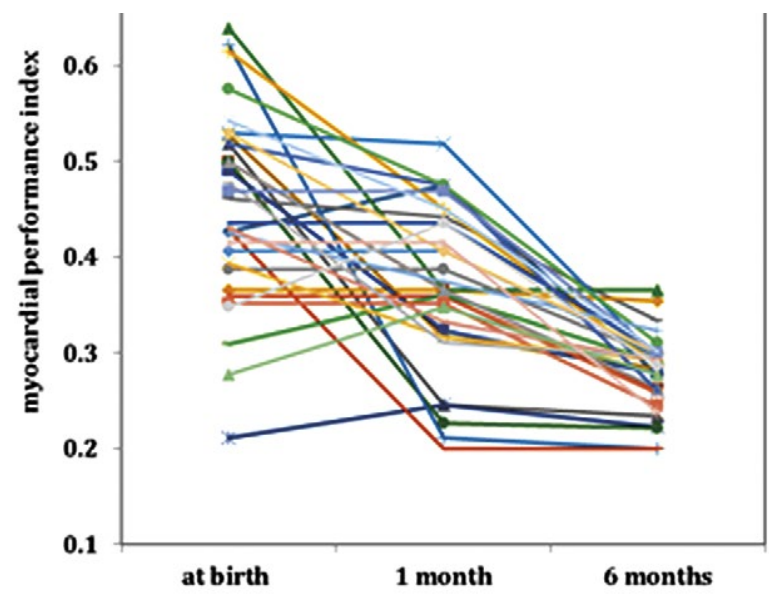

Fig 3. Evolution of the myocardial performance index over time among subjects with intrauterine grouth restriction

Moreover, there is proof that fetal and newborn cardiac and vascular malfunction could explain the increased predisposition to cardiovascular disease in childhood and adult life [11]. The intrauterine state of chronic hypoxia and undernutrition triggers a cardiac remodeling response to compensate for local stress. Postnatally, the newborn must readjust to accomodate the new demands. To satisfy the newly increased systemic pressure and decreased pulmonary pressure requires a structural remodelling of the left ventricle. The growth restricted left ven- tricle will thus be smaller in size, global mass and mass index as compared to that of AGA neonates, but these adaptive changes will not impact its systolic function. When comparing IUGR and AGA Z-scores for ventricular morphometry, we found statistically significant lower values for left ventricular parameters - LVDD in 2.5\% and LVSD in $20 \%$ of cases, which confirms up-to-date available measurements [11,28].

Certain authors have described a change in the cardiac geometry and shape of IUGR fetuses as a result of the combined pressure and possibly volume overload on the developing myocardial fibers. Instead of triggering progressive myocardial hypertrophy, in conditions of hypoxia and denutrition, the IUGR heart compensates by dilation of ventricular cavities with subsequent development of a spherical shape [11]. We did not record similar observations in our IUGR fetuses cohort, probably because evaluation was made during the transition from fetal to the postnatal circulation. However, the values of the Z-score exceeded the normal range by two standard deviations in $2(5 \%)$ IUGR neonates from our cohort.

The analysis of cardiac morphometric measurements performed at birth compared to those recorded at the six months follow-upfound significantly different dimensions in growth restricted infants. Left ventricular parameters progressively increase and "catch up" the pace respecting the standard pattern of growth reported for newborns at the one-monthand six months follow-ups.

Z-score calculations support the conclusion that there seem to be adaptative modifications in the structure of the heart in the IUGR babies. These changes persist even after birth, as shown in parameters at the one month evaluation comprised in our study. The presence of such persistent modifications was also described by other authors $[10,21]$.

There is controversy about the long term evolution of cardiac plastic changes in growth restricted fetuses. The "developmental origins of adult disease" hypothesis states that fetal cardiac programming leads to childhood or adulthood cardiovascular disease and noninsulin-dependent diabetes [26,27]. However contradictory findings have come from authors who failed to identify morphologic and functional changes in IUGR children at the 5 year follow-up $[29,30]$.

Among the Doppler parameters evaluated in our study, only the MPI was significantly higher in the IUGR group comparative to controls. The value of this index has been extensively studied in fetal populations and it has been demonstrated that for IUGR, the MPI is one of the earliest markers of cardiac malfunction, even during intrauterine life $[9,13]$. In contrast to other Doppler indices (such as pulsatility index of ductus venous and the 
aortic isthmus) the MPI remaines at increased values in fetal populations. [6,10,31].

In the adult population heart failure, the MPI is correlated with systolic dysfunction with $82 \%$ specificity and $86 \%$ sensibility at a value of $>0.47$ [31]. Our study results showed a significant association between the systolic dysfunction as reflected by the MPI and the development of IUGR. This is further evidence in support of the possible use of the MPI as a marker of cardiac disease and cardiac function progress in IUGR newborns. Serial reevaluation of cardiac morpho-functional status at a 6 month period could be performed to be able to characterize the improvement in fetal hemodynamics.

There are few reports regarding the diastolic function in children with IUGR. Altin et al [32] performed a study on neonates and infants from pregnancies with chronic fetal distressrevealing that diastolic parameters evaluated prenatally tend to normalize with at the long-term follow-up. Bjarnegard et al [33] described similar findings in young adults.

This study encompasses the first data colection which analyses echocardiographic changes in IUGR versus AGA fetuses in a Romanian population. We lack newborn population specific nomograms for echocardiographic measurements. Studies evaluating cardiac function in newborns are small-scale, there are no meta-analyses and even cohorts consist of less than 100 neonates. This is why we find our research essential for defining a clinical stand point which then needs further validation and substatiation.

There were several limiting factors which led to having such small sample sizes. On the one hand, maternal adherence to the study requirements was very low, so that we could not analyze the available data for a proper comparison of cases and controls. This was especially true for AGA babies in which we did not have enough follow-up data for the control group. Secondly, echocardiographic evaluation was performed by a single examiner, given that the procedure is delicate and time-consuming.

\section{Conclusions}

IUGR neonates undergo adaptive cardiac changes during the first six month postnatally. Left ventricle size is sensibly smaller in IUGR newborns compared to AGA newborns. The MPI is a marker of early cardiac dysfunction in newborns. IUGR neonates have smaller MPI values as a hallmark of the ongoing cardiac remodelling process. Both LV dimensions and MPI values recorded in premature, chronically distressed newborns could guide clinicans in adopting the appropriate clinical or management decisions.

\section{Acknowledgments}

This paper was published under the framework of the European Social Fund, Human Resources Development Operational Programme 2007-2013, project no. POSDRU/159/1.5/S/138776.

The authors are grateful for the support and helpful comments provided by Alexandru Zaharie and Georgiana Nemeti. The study protocol was registered on ClinicalTrials.gov (NCT02515292).

\section{Conflict of interest: none}

\section{References}

1. Sharma D, Shastri S, Farahbakhsh N, Sharma P. Intrauterine growth restriction - part 1. J Matern Fetal Neonatal Med 2016;29:3977-3987.

2. Sharma D, Farahbakhsh N, Shastri S, Sharma P. Intrauterine growth restriction - part 2. J Matern Fetal Neonatal Med 2016;29:4037-4048.

3. Easter SR, Eckert LO, Boghossian N, et al. Fetal growth restriction: Case definition \& guidelines for data collection, analysis, and presentation of immunization safety data. Vaccine 2017;35:6546-6554.

4. Vayssière C, Sentilhes L, Ego A, et al. Fetal growth restriction and intra-uterine growth restriction: guidelines for clinical practice from the French College of Gynaecologists and Obstetricians. Eur J Obstet Gynecol Reprod Biol 2015;193:10-18.

5. Crispi F, Hernandez-Andrade E, Pelsers MM, et al. Cardiac dysfunction and cell damage across clinical stages of severity in growth-restricted fetuses. Am J Obstet Gynecol 2008;199:254.e1-e8.

6. Comas M, Crispi F, Cruz-Martinez R, Figueras F, Gratacos E. Tissue Doppler echocardiographic markers of cardiac dysfunction in small-for-gestational age fetuses. Am J Obstet Gynecol 2011;205:57.e1-e6.

7. Comas M, Crispi F, Cruz-Martinez R, Martinez JM, Figueras F, Gratacós E. Usefulness of myocardial tissue Doppler vs. conventional echocardiography in the evaluation of cardiac dysfunction in early-onset intrauterine growth restriction. J Obstet Gynecol 2010;203:45. e1-e7.

8. Verburg BO, Jaddoe VW, Wladimiroff JW, Hofman A, Witteman JC, Steegers EA. Fetal hemodynamic adaptive changes related to intrauterine growth: the Generation $\mathrm{R}$ Study. Circulation 2008;117:649-659.

9. Niewiadomska-Jarosik K, Zamojska J, Zamecznik A, Wosiak A, Jarosik P, Stańczyk J. Myocardial dysfunction in children with intrauterine growth restriction: an echocardiographic study. Cardiovasc J Afr 2017;28:36-39.

10. Cruz-Lemini M, Crispi F, Valenzuela-Alcaraz B, et al. A fetal cardiovascular score to predict infant hypertension and arterial remodeling in intrauterine growth restriction. Am J Obstet Gynecol 2014;210:552.e1-e22. 
11. Crispi F, Bijnens B, Figueras F, et al. Fetal growth restriction results in remodeled and less efficient hearts in children. Circulation 2010;121:2427-2436.

12. Crispi F, Figueras F, Cruz-Lemini M, Bartrons J, Bijnens B, Gratacos E. Cardiovascular programming in children born small for gestational age and relationship with prenatal signs of severity. Am J Obstet Gynecol 2012;207:121.e1-e9.

13. Fouzas S, Karatza AA, Davlouros PA, et al. Neonatal cardiac dysfunction in intrauterine growth restriction. Pediatr Res 2014;75:651-657.

14. Akazawa Y, Hachiya A, Yamazaki S, et al. Cardiovascular Remodeling and Dysfunction Across a Range of Growth Restriction Severity in Small for Gestational Age Infants- Implications for Fetal Programming. Circ J 2016;80:2212-2220.

15. Breatnach CR, Levy PT, James AT, Franklin O, El-Khuffash A. Novel Echocardiography Methods in the Functional Assessment of the Newborn Heart. Neonatology 2016;110:248-260.

16. Bokiniec R, Własienko P, Borszewska-Kornacka MK, Madajczak D, Szymkiewicz-Dangel J. Myocardial performance index (Tei index) in term and preterm neonates during the neonatal period. Kardiol Pol 2016;74:1002-1009.

17. Kumar M, Santhanam S, Thomas N, Jana AK. A prospective observational study comparing cardiac function of small for gestational age with appropriate for gestational age babies using serial echocardiographic studies. J Matern Fetal Neonatal Med 2018;31:1-6.

18. Fenton TR, Kim JH. A systematic review and meta-analysis to revise the Fenton growth chart for preterm infants. BMC Pediatr 2013;13:59.

19. Kampmann C, Wiethoff CM, Wenzel A, et al. Normal values of $\mathrm{M}$ mode echocardiographic measurements of more than 2000 healthy infants and children in central Europe. Heart 2000;83:667-672.

20. Mertens L, Seri I, Marek J, et al. Targeted neonatal echocardiography in the neonatal intensive care unit: practice guidelines and recommendations for training. Eur J Echocardiogr 2011;12:715-736.

21. Cantinotti M, Scalese M, Murzi B, et al. Echocardiographic nomograms for ventricular, valvular and arterial dimensions in caucasian children with a special focus on neonates, infants and toddlers. J Am Soc Echocardiogr 2014;27:179-191.e2.

22. Lopez L, Colan SD, Frommelt PC, et al. Recommendations for quantification methods during the performance of a pediatric echocardiogram: a report from the Pediatric Measurements Writing Group of the American Society of Echocardiography Pediatric and Congenital Heart Disease Council. J Am Soc Echocardiogr 2010;23:465-495.

23. Hăşmăşanu MG, Bolboacă SD, Baizat MI, Drugan TC, Zaharie GC. Neonatal short-term outcomes in infants with intrauterine growth restriction. Saudi Med J 2015;36:947953.

24. Baschat AA, Odibo AO. Timing of delivery in fetal growth restriction and childhood development: Some uncertainties remain. Am J Obstet Gynecol 2011;204:2-3.

25. Alfirevic Z, Milan SJ, Livio S. Caesarean section versus vaginal delivery for preterm birth in singletons. Cochrane Database Syst Rev 2013;(9):CD000078.

26. Meyer K, Lubo Zhang. Fetal programming of cardiac function and disease. Reprod Sci 2007;14:209-216.

27. Cosmi E, Fanelli T, Visentin S, Trevisanuto D, Zanardo V. Consequences in infants that were intrauterine growth restricted. J Pregnancy 2011;2011:364381.

28. Melamed N, Yogev Y, Glezerman M. Fetal Gender and Pregnancy outcome. J Matern Fetal Neonatal Med 2010;23:338-344.

29. Mikkola K, Leipälä J, Boldt T, Fellman V. Fetal growth restriction in preterm infants and cardiovascular function at five years of age. J Pediatr 2007;151:494-499.e1-e2.

30. Morsing E, Liuba P, Fellman V, Maršál K, Brodszki J. Cardiovascular function in children born very preterm after intrauterine growth restriction with severely abnormal umbilical artery blood flow. Eur J Prev Cardiol 2014;21:12571266.

31. Cruz-Martinez R, Figueras F, Hernandez-Andrade E, Oros D, Gratacos E. Changes in myocardial performance index and aortic isthmus and ductus venosus Doppler in term, small for gestational age fetuses with normal umbilical artery pulsatility index. Ultrasound Obstet Gynecol 2011;38:400-405.

32. Altın H, Karaarslan S, Karataş Z, Alp H, Şap F, Baysal T. Evaluation of cardiac functions in term small for gestational age newborns with mild growth retardation: a serial conventional and tissue Doppler imaging echocardiographic study. Early Hum Dev 2012;88:757-764.

33. Bjarnegård N, Morsing $\mathrm{E}$, Cinthio $\mathrm{M}$, Länne $\mathrm{T}$, Brodszki J. Cardiovascular function in adulthood following intrauterine growth restriction with abnormal fetal blood flow. Ultrasound Obstet Gynecol 2013;41:177-184. 